\title{
Risk Limiting Dispatch With Optimal Curtailing in Active Distribution Networks
}

\author{
D. Georgiev and E. Janeček
}

\begin{abstract}
Renewable energy sources often provide intermittent power at distributed locations in transmission and distribution networks. Efficient utilization of these sources must consider economics, computation, and reliability in managing network resources. A new computational tool for efficient dispatch of intermittent sources is developed using the risk limiting operational paradigm. Optimal dispatch of regulation and load following ancillary services is computed using current estimates of future random energy production. Substitution of intermittent power with firm power through a curtailment strategy is used to avoid regulation costs in excess of current firm power prices. The underlying mathematical framework is a non-convex optimal power flow problem, which is shown to have an exact convex relaxation under a set of realistic assumptions. The methodology is successfully tested on an IEEE 30-bus test system. Several operational effects of source uncertainty are captured. For instance, optimal solutions are shown to create power flows that mutually compensate intermittencies from different sources to minimize regulation requirements.
\end{abstract}

\section{INTRODUCTION}

In the global effort to curb carbon emissions, governments have proposed many ambitious renewable energy utilization targets [1]. Renewable energy sources (RESs) are often intermittent [2], [3] and geographically distributed at locations with the greatest energy potential. To utilize this energy at the penetration levels proposed, active distribution networks (ADNs) permitting dynamic rerouting of power flows are envisioned to replace their passive predecessors. In this area, much attention has been given to advanced metering technologies and the demand side management they enable. Smart management of the energy sources has received less attention. Government incentives in the form of feed-in tariffs (promise to buy all renewable energy generated for the next 5-10 years at fixed prices) significantly decrease the flexibility of distributed source management. Herein, we increase the flexibility by integrating two control tools, curtailing and scheduling, in a risk limiting dispatch method.

Curtailing of intermittent sources (ISs) has been explored in literature as a tool for increasing system safety [1], [4], [5]. Curtailment as a last resort tool for maintaining acceptable voltage levels is explored in [1] and fixed curtailment in scheduling problems is considered in [5]. The study in [4] applies optimal power flow tools to historical data in order

This work was supported by the European Regional Development Fund (ERDF), project "NTIS New Technologies for the Information Society", European Centre of Excellence, CZ.1.05/1.1.00/02.0090 and by the grant TA CR TA01020865.

D. Georgiev and E. Janeček are with the Department of Cybernetics in the Faculty of Applied Sciences, University of West Bohemia in Pilsen, Pilsen 30614, Czech Republic. georgiev@kky.zcu.cz, janecek@kky.zcu.cz to estimate the network maximum capacity for intermittency. To the best of our knowledge, no work considers optimizing curtailing policies as a complementary tool to scheduling firm power sources while respecting feed-in tariffs.

The concept of risk limiting dispatch has only recently been proposed [6]. In distribution systems there are several layers of response that utilize a priori allocated generation capacity to balance power deviations (e.g., the spinning reserves, supplemental reserves, and replacement reserves). In [6] the resources are allocated using the latest predictions to actively manage the risk at the distribution level. Other risk conscious dispatch methods have been proposed [5], [7]. These build on dynamic programming principles. It is not clear how suitable they are for real time applications at the time scale of minutes operating on networks comprising hundreds of thousands of nodes. Curtailing optimization is not considered in any of the above cited work.

Herein we consider curtailment from the economical perspective as a complementary tool to traditional power allocation. The underlying idea is energy that costs more to regulate than to replace should be replaced even if it has already been purchased (as is the case with feed-in tariffs). The network is considered at two time instances representing two time-scales of real-time market operations. At the first time instance (usually at the hour-ahead timescale), ISs forecasts are used to manually allocate the load following sources (LFSs), curtailing policies of the ISs are fixed, and regulatory power sources (RPSs) are reserved. At the second time instance (usually at the minute-ahead timescale), the loads are realized and the power from the ISs is generated and curtailed according to the policies computed at the first time instance. At this time the reserved regulatory power is automatically dispatched as needed to balance the minuteto-minute fluctuations.

The innovation is to consider economic benefits of curtailment within the feed-in tariff framework by capitalizing on potential regulation savings within the hour markets. This advances existing work considering curtailment without feed-in tariffs (e.g., see [8]) or last minute curtailment [1]. The time scale of operation for the tool is on the order of tens of minutes, consistent with the time scale of present load following and regulation ancillary services [9]. Hence computational efficiency is stressed throughout.

The remainder of the paper is organized as follows: The network model with the necessary assumptions is presented in Section II. The new main optimization problem is presented in Section III and further formulated into a convex problem in Section IV. Longer derivations are saved for 
the appendix. The IEEE 30-bus system study is presented Section V. The paper concludes in Section VI.

\section{NeTWORK MODEL}

The following notation is used throughout. Capital letters are used to denote random variables and matrices. The letter $i$ is reserved for the imaginary unit. Any vector $\left(x_{1}, \ldots, x_{n}\right)$ can be simply written as $\left(x_{k}\right)_{k \in\{1, \ldots, n\}}$ or as $x$. For random variables $X$, the expectation value is written as $\langle X\rangle$ or simply as $\mu_{X}$. The variance is written as $\langle\langle X\rangle\rangle$ or simply as $\sigma_{X}$. Correlations between two random variables $X$ and $Y$ are simple denoted by $\rho_{X, Y}$ Variables are represented in the dimensionless per unit scale unless specified otherwise, in which case the superscript $p u$ is used.

A two time step operation is assumed. At time $t-\delta$, the latest prediction of the network for the time $t$ is considered. LFSs and RPSs are allocated along with curtailment policies for the ISs. At time $t$, intermittent power generation is realized and curtailed and the corresponding regulatory response is dispatched.

The prediction of the network is described by a directed graph where each node corresponds to a load, a generator, or a bus and each branch corresponds to a power line or a transformer where the direction of the branch represents the direction of the power flow. The set of nodes is given by the finite set $\mathcal{N}=0,1, \ldots, N$ and the set of branches is defined by the set $\mathcal{B} \subset \mathcal{N} \times \mathcal{N}$, where each $(k, m) \in \mathcal{B}$ is directed from node $k$ to node $m$. For each node $k \in$ $\mathcal{N}$, the set of outgoing branches is given by the set $\mathcal{O}_{k}=$ $\{b \in \mathcal{B} \mid b=(k, j), j \in \mathcal{N}\}$ and the set of incoming branches is given by the set $\mathcal{J}_{k}=\{b \in \mathcal{B} \mid b=(j, k), j \in \mathcal{N}\}$. If there exists a path from node $k$ to node $m$, then $k$ is said to be upstream of $m$ and $m$ is said to be downstream of $k^{1}$.

The predicted state of the network is described by a set of random variables assigned to the nodes and branches. At each node $k \in \mathcal{N}$, is the phase $\Theta(k)$, the load $p_{L}(k)$, the generated load-following power $p_{G}(k)$, the regulatory power $P_{R}(k)$, and the intermittent power $W(k)$. At each branch $b \in \mathcal{B}$, is the impedance $z(b)=r(b)+i x(b)$, the power loss $L(b)$, and the power transmitted (in the direction of the branch) $F(b)$. Throughout most of the paper, only active powers are considered.

The following list of assumptions is commonly used.

A1. The power flows only in the edge direction,

A2. the network topology and branch impedances are fixed and known, the network is balanced and operating under normal conditions with no failures and filled power demands,

A3. the loads $p_{L}$ are known,

A4. the voltage drop is negligible, i.e., for all $k \in \mathcal{N}$, the maghe nitude of the node voltage is $v_{0}$,

A5. the probability of a large voltage phase deviation is small, i.e., for all nodes $k \in \mathcal{N}$, if the probability of $\left\{\left|\Theta_{k}-\left\langle\Theta_{k}\right\rangle\right|<\epsilon\right\}$ is approximately one.

\footnotetext{
${ }^{1}$ In optimal power flow solutions, cycles are automatically avoided making this notation meaningful.
}

Assumption 1 is important to the stochastic formulation and cannot be easily removed (e.g., by splitting the power into a positive and negative component). A sufficient algorithm for computing the flow direction is given in Section IVF. Assumption 2 is required for standard physical laws to apply. Assumptions 3-5 are common in the DC OPF formulation [10].

\section{OPTIMIZATION}

Computations in the proposed framework take place at two separate time instances. LFSs, curtailment policies, and RPSs are allocated first. The regulatory response is computed second at the same time the loads and the ISs are realized. Optimization of the dispatch and curtailment policies takes place in the reverse order. In this section, we assume the policies by which regulation power is dispatched using latest measurements (e.g., FACTS or SVC control policies) are fixed and present only in the second optimization problem. In the next section we revisit the regulation axiomatically and solve for the response.

This approach simplifies the optimization but does not oversimplify it. The RPSs are dispatched when all contingency information is known. This includes a diverse spectrum of phenomena including blackouts, generator failures, load changes, transient effects, etc.. The $N-1$-condition is used in practice to allocate RPSs. The actual response is computed using only local information by preprogrammed devices distributed in the network [6]. The complexity of this response makes it difficult to characterize its statistics. Hence, considering a specific regulation policy when allocating the other resources can increase the computational efficiency (possibly by a great margin if convexity cannot be maintained) and increase the lag time between measurement and implementation without yielding an easily verifiable benefit in performance.

A solution to the following optimization problem allocates the LFSs and computes the curtailment policies for the ISs.

\section{Problem (PFC).}

$$
\underset{p_{G}, P_{R}, W_{C}}{\operatorname{minimize}}\left\langle J\left(p_{G}, P_{R}, W_{C}\right)\right\rangle,
$$

subject to, for all $k \in \mathcal{N}$ and $b \in \mathcal{B}$,

$$
\begin{aligned}
p_{G}^{-}(k) & \leq p_{G}(k) \leq p_{G}^{+}(k),\left\langle P_{R}^{2}(k)\right\rangle \leq p_{R}^{+}(k), \\
0 & \leq\langle F(b)\rangle,\left\langle F(b)^{2}\right\rangle \leq f^{+}(b), \\
\langle D(k)\rangle & =\left\langle S(k)-\sum_{I_{k}} L(k)\right\rangle,\langle\langle D(k)\rangle\rangle=\langle\langle S(k)\rangle\rangle,
\end{aligned}
$$

where $W_{C}$ is the dispatched intermittent power, $F$ is the vector of line flows, $L$ is the vector of line losses, and $S$ and $D$ are the vectors of flows into and out of each node, i.e.,

$$
\begin{aligned}
& S(k)=p_{G}(k)+W_{C}(k)+\sum_{b \in \mathcal{J}_{k}} F(b), \\
& D(k)=p_{D}(k)+\sum_{b \in \mathcal{O}_{k}} F(b)+P_{R}(k) .
\end{aligned}
$$


In the above formulation, the expectation of the node power balance is used. This is consistent with the statistical information usually available for physical systems. The second moment line flow and power flow constraints are introduced to limit risk and hence are referred to in the sequel as risk limiting constraints. These are new to the optimal power flow framework. The line constraints $\left(\left\langle F(b)^{2}\right\rangle \leq\right.$ $\left.f^{+}(b)\right)$ ensure branches aren't overloaded. The non negative constraint on the power flows is given by Assumption 1 and included here for clarity. The power flow constraints $(\langle\langle D(k)\rangle\rangle=\langle\langle S(k)\rangle\rangle)$ ensure the regulation capacity is sufficient to compensate for the intermittent generation. Omitting losses from this expression increases the variation of the flow exiting a node and hence overestimates the required downstream regulation.

Problem PFC in this form is non-convex. The reformulation of Problem PFC into a computationally tractable, convex problem is described next. Part of the reformulation is choosing models of intermittency and regulation that lend themselves to efficient computation. This modeling is addressed first. Second the assumptions are used to derive formulas for the different constraints in Problem PFC. Third a convex relaxation is introduced and conditions for its exactness are presented. Finally a sufficient algorithm is proposed for the computation of the branch flows.

\section{SOLUTION}

Problem PFC is reformulated below into a convex optimization problem. The large scale of distribution networks (with possibly millions of customers) makes convexity the minimum requirement for any reasonable optimization tool.

\section{A. Intermittency}

Curtailment of each intermittent power source is considered separately to allow for sources at undesirable locations on the network to be regulated independently of conveniently located sources. The dispatched intermittent power at node $k \in \mathcal{N}$ has the form

$$
W_{C}(k)=\min (\lambda(k), W(k)),
$$

where $\lambda$ is the vector of curtailment thresholds placed on the sources to limit the uncertainty they inject into the network. The distribution of the generation capacity $W$ depends on the particular source. Stochastic models of intermittent power sources are an active area of research. The set of distributions that can be handled within the framework of Problem PFC in a convex way is constrained due to the curtailing policies. For the sake of simplicity, the sources are approximated with binomial distributions. For every $k \in \mathcal{N}$, assume the generation capacity $W(k)$ is described by

$$
W(k)= \begin{cases}w^{+}(k), & \text { with prob. } q(k), \\ w^{-}(k), & \text { with prob. } 1-q(k) .\end{cases}
$$

The methodology presented herein is extendable to a greater set of distributions, e.g., the set of distributions with nonincreasing probability densities above the minimum permissible curtailment threshold $\lambda^{-}$. The resulting optimization problems, however, are only quasi-convex.
We also assume that for all $k, m \in \mathcal{N}$, the correlation of $W(k)$ and $W(m)$ is equal to zero. This assumption leads to a modular system description is exploited in the optimal solution to lower regulation costs by transmitting intermittent power flows over common branches. This generalizes the grouping strategy for regulating intermittencies [9]. Composite system models or parametrisations (e.g., see [11]) can be used to introduce correlations.

\section{B. Regulation and Risk}

The RPSs are dispatched using automatic generation control once all contingencies are known. The broad spectrum of contingencies make it difficult to statistically predict the response. Instead we approach this problem axiomatically.

Axiom 1 (Regulation). The regulatory response satisfies the following conditions:

1) There is a unique path from each intermittent source to each regulatory power source,

2) the regulatory response minimizes the squared norm $\left\|\sigma_{F}\right\|^{2}$ of the uncertain part of the power flows under the constraints of Problem PFC, and

3) the regulatory response has a zero mean.

Out of these axioms, only Axiom 2 is atypical. The assumption is that under normal operating conditions the power flows optimally accommodate the electricity demand, satisfy line limitations, and produce prescribed node voltages. Hence, the automated responses of passive and active network elements act to minimise the deviation of the power flows from their nominal values.

Together these conditions lead to a simple expression for the following risk limiting constraint of Problem PFC

$$
\langle\langle D(k)\rangle\rangle=\langle\langle S(k)\rangle\rangle .
$$

Since the ISs are assumed to be independent, the first condition implies the flows into any node are uncorrelated. The second condition leads to an explicit expression for correlation of the flows exiting any node. Starting at any node $k \in \mathcal{N}$ with no incoming branches it follows

$$
\begin{aligned}
& \left\|\left(\sigma_{F(b)}\right)_{b \in \mathcal{O}_{k}}\right\|^{2}=\sigma_{W_{C}(k)}^{2}-\sigma_{P_{R}(k)}^{2} \\
& -\sum_{a, b \in \mathcal{O}_{k}, a \neq b} \min _{\rho_{F(a), F(b)}}\left\{\rho_{F(a), F(b)} \sigma_{F(a)} \sigma_{F(b)}\right\} . \\
& -\sum_{a \in \mathcal{O}_{k}} \min _{\rho_{F(a), R(k)}}\left\{\rho_{F(a), R(k)} \sigma_{F(a)} \sigma_{P_{R}(k)}\right\}
\end{aligned} .
$$

Hence, for all $a, b \in \mathcal{O}_{k}$, the correlations $\rho_{F(a), F(b)}$ and $\rho_{F(a), R(k)}$ are equal to 1 . Since the path from any IS to any RPS is unique, we can proceed by induction to show that these correlations are equal to 1 at all nodes.

By these axioms, the risk limiting constraint has the form

$$
\sum_{b \in \mathcal{O}_{k}} \sigma_{F(b)}+\sigma_{P_{R}(k)}=\sqrt{\left\|\left(\sigma_{F(b)}\right)_{b \in \mathcal{J}_{k}}\right\|^{2}+\sigma_{G(k)}^{2}} .
$$

This expression allows a distributed description of the optimization problem. At each node, knowledge of upstream and downstream fluctuations is not necessary to formulate the constraints. There is a caveat. In order for the first axiom to be satisfied, the flow directions must be known. This is 
the primary reason for Assumption 1. If the directions were not known and flows were instead separated into positive and negative components, then this expression would give an incentive to route flows in cycles.

\section{DC Assumption}

The DC OPF formulation is computationally tractable and has proven economic value (i.e., used in practice to minimize energy costs). This suggests the formulation achieves the necessary balance between computational simplicity and modeling accuracy. For these reasons, we choose to build Problem PFC on the same set of physical assumptions.

The standard DC OPF problem [12] is derived under Assumptions 2- 5. Moreover, it is assumed that all resistances are zero and all reactive loads and generation are zero [10]. This yields a linear expression for the expected line flows in terms of the expected phase angles, for all $b \in \mathcal{B}$

$$
f(b)=\frac{1}{x(b)}(\theta(b(2))-\theta(b(1)))
$$

A slack bus is arbitrarily chosen to be the first bus and assigned the null phase.

These assumptions alone yield the lossless DC OPF problem. Losses are added to provide a locational context. Commonly used linear loss models (e.g., see [13]) fail to capture uncertainty effects. Consider a line $b \in \mathcal{B}$ with the current $I$. By the Jensen's inequality, the expected losses across the line satisfy

$$
\langle L(b)\rangle=r(b)\langle\|I\|\rangle \geq r(b)\|\langle I\rangle\| .
$$

Hence, linear losses underestimate intermittent losses. Instead we develop a more realistic quadratic loss model.

On a physical scale (W, MW, etc.), for any $b \in \mathcal{B}$, the power flowing into the line satisfies $\|F(b)\|=\|V\|\|I\|$, where $V$ is the voltage at the line's origin. Solving for the current yields

$$
L(b)=r(b) \frac{\|F(b)\|}{\|V\|} .
$$

By Assumptions 4 and 5, the voltage norm $\|V\|=\left\|v_{0}\right\|$ and the power flow is real. In the per unit scale, where the voltage scaling factors are related by $p_{0}=v_{0}^{2} / r_{0}$, the expression becomes

$$
L^{p u}(b)=r^{p u}(b)\left(F^{p u}(b)\right)^{2}
$$

Finally, there are two options in appending the losses to the power balance equations. Losses can be subtracted from either the flows exiting the node or from the flows entering the node. Each option can produce much different results. Subtracting losses from outgoing flows implies all flows $F(b)$ represent the power exiting a line, which is less than the power entering a line and thereby produces smaller losses (infinite power can be transmitted across a line in this scenario). For this reason, losses are subtracted from incoming flows and all flows are assumed to be positive.

\section{Cost}

The costs attributed to RESs and traditional power sources are fundamentally different. Traditional power is purchased for economical and safety reasons, while (in many areas across Europe and the US) public policy sets feed-in tariffs that determine what percentage of the market price all generated renewable energy is to be purchased.

The cost function $J$ in Problem PFC is chosen to 1) penalize purchases of power from LFSs and RPSs, 2) promote use of intermittent power, and 3) penalize system inefficiencies, i.e., line losses. Specifically, the cost function has the following form

$$
\begin{aligned}
J\left(p_{G}, P_{R}, W_{C}, L\right) & =p_{G}^{T}\left(a_{G}+B_{G} p_{G}\right) \\
& +P_{R}^{T}\left(a_{R}+B_{R} P_{R}\right) \\
& +\left(W-W_{C}\right)^{T}\left(a_{W}+B_{W}\left(W-W_{C}\right)\right) \\
& +L^{T} a_{L},
\end{aligned}
$$

where the matrices $B_{G}, B_{R}$, and $B_{W}$ are assumed to be diagonal and positive definite and the vectors $a_{G}$ and $a_{L}$ are assumed to be positive.

\section{E. Convex Relaxation}

Problem PFC with its constraints and cost as specified in the previous sections is not convex due to the quadratic losses and risk limiting constraints. Relaxing the equality constraints generates the following simple convex relaxation. The proposed dispatch and curtailing methodology is built on solving this problem.

\section{Problem (Co-PFC).}

$$
\begin{aligned}
& \begin{array}{ll}
\underset{p_{G}, \sigma_{R}, \lambda,}{\operatorname{minimize}} & p_{G}^{T}\left(a_{G}+B_{G} p_{G}\right)+\sigma_{R}^{T} B_{R} \sigma_{R}+\mu_{L}^{T} a_{L} \\
\mu_{\left(W-W_{C}\right)}+\sigma_{\left(W-W_{C}\right)}^{T} B_{W} \sigma_{\left(W-W_{C}\right)},
\end{array} \\
& \mu_{\left(W-W_{C}\right)}, \sigma_{\left(W-W_{C}\right)}, \\
& \mu_{F}, \sigma_{F}, \mu_{\Theta}, \mu_{L}
\end{aligned}
$$

subject to, for all $k \in \mathcal{N}$ and $b \in \mathcal{B}$,

$$
\begin{aligned}
p_{G}^{-}(k) & \leq p_{G}(k) \leq p_{G}^{+}(k), \sigma_{P_{R}(k)}^{2} \leq p_{R}^{+}(k), \\
0 & \leq \mu_{F(b)}, \mu_{F(b)}^{2}+\sigma_{F(b)}^{2} \leq f^{+}(b), \\
\mu_{W_{C}(k)} & =q(k)\left(\lambda(k)-w^{-}(k)\right)+w^{-}(k), \\
\sigma_{W_{C}(k)} & =\sqrt{q(k)(1-q(k))}\left(\lambda(k)-w^{-}(k)\right) \\
\mu_{\left(W(k)-W_{C}(k)\right)} & =q(k)\left(w^{+}(k)-\lambda(k)\right), \\
\sigma_{\left(W(k)-W_{C}(k)\right)} & =\sqrt{q(k)}\left(w^{+}(k)-\lambda(k)\right) \\
\sum_{b \in \mathcal{O}_{k}} \mu_{F(b)}+p_{L}(k) & =p_{G}(k)+\mu_{W_{C}(k)} \\
& +\sum_{b \in I_{k}}\left(\mu_{F(b)}-\mu_{L(b)}\right), \\
\sum_{b \in \mathcal{O}_{k}} \sigma_{F}(b)+\sigma_{R}(k) & \geq \sqrt{\left\|\left(\sigma_{F}\right)_{b \in \mathcal{J}_{k}}\right\|^{2}+\sigma_{G}(k)^{2}}, \\
\mu_{L(k)} & \geq r(k)\left(\mu_{F(k)}^{2}+\sigma_{F(k)}^{2}\right) .
\end{aligned}
$$

Problem Co-PFC is a convex optimization problem with a positive semidefinite quadratic cost function, quadratic constraints, and second order cone constraints. It is solvable 
by readily available tools such as the freeware tool cvx for Matlab.

Last two constraints in Problem Co-PFC are the convex relaxations. For these relaxation to be exact, the inequalities must be equalities at the optimal point. The following theorem gives the conditions when this is true. The proof can be found in the appendix.

Theorem 1. Consider the introduced network and generator models and suppose the Regulation Axiom and Assumptions 1- 5 hold. If it is optimal to utilize the full intermittent generator capacity $W$ when no intermittency is present (i.e., when $q=1$ ), then, for all values of $q$, any solution to Problem Co-PFC generates variables $p_{G}, P_{R}, W, F$, and $L$ that solve Problem PFC.

Unlike other more general relaxations explored in literature (e.g., see [14]), the above relaxation is independent of the network topology. Intuitively, artificially increasing the losses $L$ enables the intermittent sources to transmit unused capacity and thereby avoid curtailing penalties. However, if full capacity is used when $q=1$, then any curtailing for other values of $q$ is done to limit regulation costs or congestion, neither of which can be alleviated by increasing losses.

\section{F. Flow Direction Algorithm}

Assumption 1 states that the flow directions are known. Below we use the following algorithm to compute the flow directions. The algorithm correctly finds the optimal flow directions if it terminates successfully (see the Appendix). The Flow Orientation (FO) algorithm terminated successfully in all of the following cases, however, exceptions were found. The finding of an algorithm that necessarily terminates successfully is an open problem.

Algorithm (Flow orientation). Define a relaxed Co-PFC problem where the flows $F$ are allowed to be negative.

1) Select an arbitrary initial flow direction.

2) Solve the free Co-PFC problem.

a) If all flows are positive, stop.

b) Otherwise, locate all edges where the flows are negative, reverse their orientation, and repeat this step.

\section{CAse Study}

Problem PFC was tested on a modified IEEE 30-bus network first published in [15] and adapted for economic studies in [16]. For the network topology, see Figure 3. Loads and load following generators were placed as in [16] with the same capacities. The generators were also given unlimited spare capacity for regulation. No other means of regulation were considered. Locations of the intermittent sources are shown in Figure 3 and their parameters are listed in Table I. Cost coefficients for the load following generators are equal to those of the intermittent sources. Cost coefficients of the regulatory sources were defined to be 100 times larger. In practice, the prices of regulatory power are only about 10 times larger. The excessive penalty was selected to emphasize the effects of uncertainty in the test network. Limits on branch flows were ignored in this study to also help isolate uncertainty effects. Various values for the intermittency probability vector $q$ were tested.

\section{TABLE I: Intermittent Power Sources}

\begin{tabular}{c|c|c|c|c} 
Node & $w^{+}[M W]$ & $w^{-} / w^{+}$ & $a_{W}$ & $B_{W}$ \\
\hline 24 & 16 & .75 & .023 & 2.7 \\
25 & 16 & .75 & .023 & 2.7 \\
21 & 8 & .75 & .023 & 2.7 \\
15 & 10 & .75 & .023 & 2.7 \\
12 & 6 & .75 & .023 & 2.7 \\
3 & 11 & .75 & .023 & 2.7
\end{tabular}

\section{A. Results and Discussion}

Problem Co-PFC was solved for the probabilities $q \in$ $\{0.2,0.4,0.5,0.6,0.7,0.8,0.9,1.0\}$. The results are summarized in Figures 1, 2, and 3 where the base power of $100 M V A$ was used to convert the results into the appropriate physical units.

The following results were expected. For the nominal case $(q=1)$, the load following generators are used the least and priority is given to the intermittent sources. Also the full capacity (i.e., zero curtailment) is used implying Theorem 1 holds and the convex relaxation is exact. As the probability of maximum capacity decreases, all generators that have spare capacity increase production and regulatory allocations also increase.

The following are some further observations made regarding curtailment strategies. Some ISs are at locations where regulation is prevented by the flow directions, e.g., at node 21. At these locations full curtailment is used in the presence of any uncertainty. At other locations, where regulation is possible, curtailment is used below a certain threshold on $q$, see Figure 2. Below this threshold, curtailment is nonincreasing with decreasing $q$. This is despite the variance of the intermittent power reaching its maximum at $q=.5$. The value of this threshold seems to depend on the interconnectivity at the location as well as on the local power demand.

The following are some observations made regarding regulation. Even though regulation increases in general as uncertainty increases, maximum regulation is not allocated at $q=.6$ instead of $q=.5$, which is when the variance of $W$ is the greatest. The point of maximum regulation seems to be determined by the curtailment strategy. The optimal solution also routs flows from different ISs along mutual branches to exploit their statistical independence for regulation reduction. This alleviation in regulation requirements corresponds to less stringent curtailment strategies. For instance, the generators at nodes 3, 12, and 15 all balance each other at node 4 before regulation is employed at node 2 . These three generators use 3 of the 4 least stringent curtailment policies. Moreover, generator 12 is never curtailed despite having one of the larger capacities, which corresponds to greater uncertainty injected into the network. 

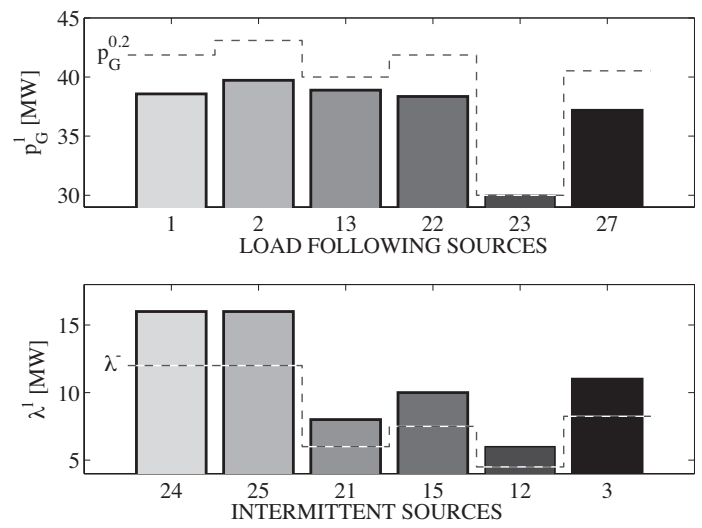

Fig. 1: Dispatch of LFPs for the $q=1$ scenario and IS capacity. The dashed line in the top plot illustrates the generation at $q=.2$ when the most additional power is required. The dashed line in the bottom plot illustrates the minimum power to which the ISs can be curtailed.

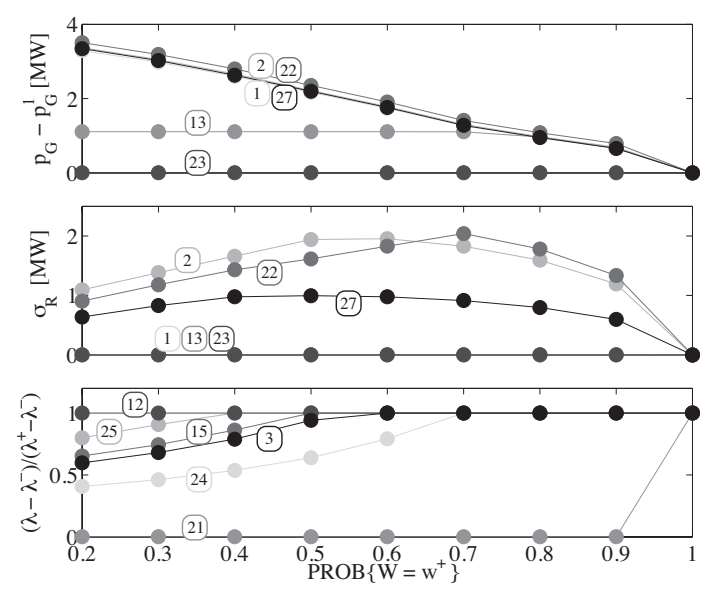

Fig. 2: Changes in allocation of the LFSs and RPSs, and in curtailment of the ISs. The top plot shows the changes in LFS allocations from the nominal value $P_{G}^{1}$. The middle plot shows the RPS allocation. The bottom plot shows the relative changes in curtailment as a percentage of the maximum curtailment. Node numbers are indicated adjacent to their corresponding graph.

\section{B. An Open Problem}

The case study generated the following open problem regarding the Regulation Axiom. The axiom is built on the minimization of flow variances under the constraint

$$
\langle\langle D\rangle\rangle=\langle\langle S\rangle\rangle,
$$

where regulation is on the left hand side of the equation as part of the variable $D$. By choosing sides, correlations between the regulatory response and upstream intermittency (the right hand side) is effectively ignored. This forces all

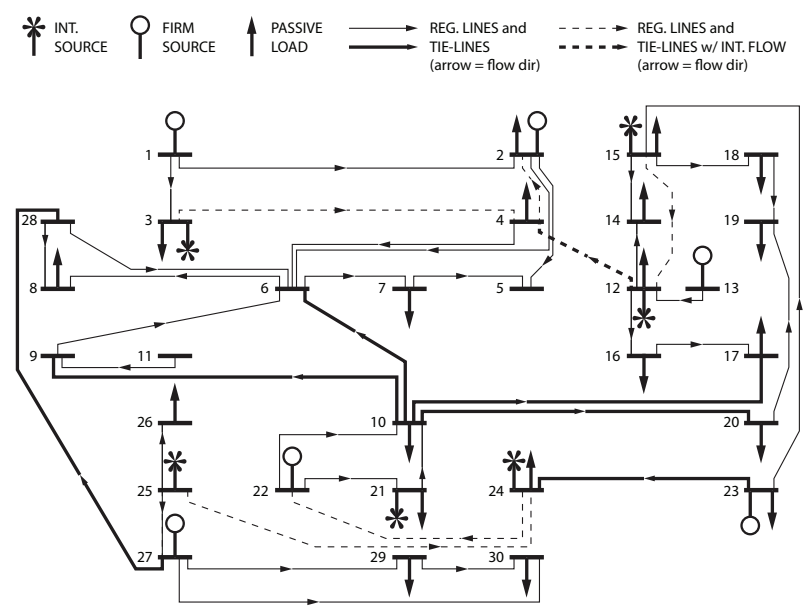

Fig. 3: The IEEE 30-bus test system showing the flow direction (same for all considered values of $q$ ). Dashed lines indicate intermittent flow.

sources to route their intermittency to downstream regulatory nodes. As a result, some nodes (e.g., node 21) cannot tolerate any uncertainty even though a regulatory node is nearby (e.g., node 22). Such nodes then fully curtail their sources, which may be inefficient. The axiomatic approach proposed herein seems to be valuable in formulating a useful and tractable optimization method and further study on the formulation of the optimality criterion in this axiom is one way of improving this method.

\section{CONCLUSION}

Curtailment and source allocation are integrated in a twostep optimization problem to create a new risk limiting tool. To tool is suitable for fast computation in large networks. The underlying optimization problem is convex and has a distributed description. The tool is also potentially applicable in networks with a high degree of renewable energy penetration. Risk limiting constraints were introduced to manage risks of congestion and power imbalances. Finally, the tool minimizes energy costs. Curtailment was considered under the feed-in tariff and therefore employed only when regulation of intermittent power were more expensive than its replacement.

\section{APPENDIX}

\section{A. Exactness of the convex relaxation (Theorem 1)}

Recall the Regulation Axiom prevents cycles in the path from any IS to any RPS. Moreover, by definition, the matrices $B_{G}, B_{R}$, and $B_{W}$ are positive definite and the vectors $a_{G}$ and $b_{W}$ are positive.

Proof: The two relaxed constraints are:

$$
\begin{array}{ccc}
C 1: & \sum_{b \in \mathcal{O}_{k}} \sigma_{F(b)} \geq \sqrt{\left\|\left(\sigma_{F(b)}\right)_{b \in \mathcal{J}_{k}}\right\|^{2}+\sigma_{W(k)}^{2}}-\sigma_{P_{R}(k)}, \\
C 2: & \mu_{L(b)} \geq r(b)\left(\mu_{F(b)}^{2}+\sigma_{F(b)}^{2}\right) ;
\end{array}
$$


To show that $C 2$ is an equality at the optimal solution $\xi^{o}$, simply consider an edge $b \in \mathcal{B}$ where $C 2$ is strict by a margin of $\delta$. The flow $F(b)$ is necessarily positive. Find all trees in the network that contain this flow. If in any of these trees there exists a node $k \in \mathcal{N}$ with $\mu_{G(k)}>0$. Then simply lower $L(b)$ and $\mu_{G(k)}$ to produce a lower cost. If no such node exists, consider the optimal solution $\xi^{1}$ when the probability $q=1$. There must be an upstream node $k_{1}$ where $\mu_{W\left(k_{1}\right)}>$ $\epsilon, \delta>\epsilon$, and where the outgoing flow $\mu_{F(a)}$ in the direction of $b$ is greater by $\epsilon_{1}$ than in $\xi^{1}$ (otherwise the downstream demand would not be met). This implies there exists another node $k_{2}$ that is compensating for the $\epsilon_{1}$ difference (recall in the solution $\xi^{1}$ the full capacity $W$ is used). If $\mu_{G\left(k_{2}\right)}>\epsilon_{2}$, lower the generation by $\epsilon_{2}$ and re-route the flows. Otherwise, proceed in this fashion until one such node is found (such node must exist since the intermittent sources cannot meet the demand and waste power). This can be done without exceeding branch capacities since the network can handle the full intermittent power and only mean flows are being re-routed.

We now address $C 1$. Start with a node $k \in \mathcal{N}$ with $\left(\sigma_{F(k)}\right)_{k \in \mathcal{O}_{k}}=0$ and $\left(\sigma_{F(k)}\right)_{k \in \mathcal{J}_{k}}=0$. Suppose $C 1$ is strict at the optimal solution $\xi^{o}$. Then $\sigma_{P_{R}(k)}$ must be positive. Consider the alternate solution $\xi^{\prime}$ that is everywhere the same as $\xi^{o}$ except for $\sigma_{P_{R}(k)}^{\prime}=0$. This solution is admissible and yields a lower cost contradicting that $\xi^{o}$ is optimal.

The proof continues by induction. Consider a node $k \in \mathcal{N}$ with $\left(\sigma_{F(b)}\right)_{b \in \mathcal{O}_{k}}=0$ and, for some $a \in \mathcal{J}_{k}, \sigma_{F(a)}>0$. The standard deviation $\sigma_{P_{R}(k)}$ must be positive. Suppose $C 1$ is strict at the optimal solution $\xi^{o}$. Consider the alternate solution $\xi^{\prime}$ that is everywhere the same as $\xi^{o}$ except for $\sigma_{P_{R}(k)}$, which is replaced with some admissible $\sigma_{P_{R}(k)}^{\prime}<$ $\sigma_{P_{R}(k)}$. This solution yields a lower cost contradicting that $\xi^{o}$ is optimal.

For the induction step, take a node $k \in \mathcal{N}$ with, for some $b \in \mathcal{O}_{k}, \sigma_{F(b)}>0$. Suppose $C 1$ is strict at the optimal solution $\xi^{o}$. There necessarily exists another node $m \in \mathcal{N}$ with $\left(\sigma_{F(a)}\right)_{a \in \mathcal{O}_{m}}=0$ and a sequence of edges $\left\{b, b_{1} \ldots, b_{n}\right\}$ leading from $k$ to $m$ for which $\sigma_{F\left(b_{\ell}\right)}>0$, for all $\ell \in\{1, \ldots, m\}$. Consider the alternate solution $\xi^{\prime}$ that is the same as $\xi^{o}$ with the exception of $\sigma_{F(b)}$ and $\sigma_{F\left(b_{\ell}\right)}, \ell \in$ $\{1, \ldots, m\}$, which are replaced by some admissible $\sigma_{F(b)}^{\prime}<$ $\sigma_{F(b)}$ and $\sigma_{F\left(b_{\ell}\right)}, \ell \in\{1, \ldots, m\}<\sigma_{F\left(b_{\ell}\right)}, \ell \in\{1, \ldots, m\}$. This solution yields a cost that is at most equal to the optimal cost. Moreover $C 1$ at node $m$ is strict. By previous results, this contradicts that $\xi^{o}$ is optimal.

\section{B. Flow orientation algorithm}

In this section we show that the FO algorithm introduced in Section V is sufficient. Finding an algorithm that is also necessary is part of the future work.

Proof: Consider the undirected problem (UP) with the cost $J^{u}(\xi, d)$ defined over all optimization variables $\xi$ as well as edge directions $d$. Consider also the free problem (FP) with the cost $J^{f}\left(\xi, d_{0}\right)$ that has specified edge directions $d_{0}$ and unrestricted flow directions (i.e., the variables $\mu_{F(b)}$ are allowed to be negative). Compare now the optimal costs of the two problems. For any optimal solution $\xi^{u}$ to the UP construct a related solution $\xi^{f}$ by fixing the flows, for all $b \in \mathcal{B}$,

$$
\mu_{F(b)}^{f}=\left\{\begin{array}{cc}
\mu_{F(b)}^{u}, & \text { if } d^{u}(b)=d^{0}(b) \\
-\mu_{F(b)}^{u}, & \text { otherwise. }
\end{array}\right.
$$

If the flows $\mu_{F(b)}^{f}$ are all nonnegative, then the costs are equal (without loss of generality we can assume that $\mu_{F(b)}$ is always greater than $\left.\mu_{L(b)}\right)$. If some flows are negative, however, the losses associated with the negative flows can be shown to be larger in the UP. Hence, the FP cost is always equal to or less than the UP cost.

The orientation algorithm instructions say to stop when the flows in the FP are nonnegative. The resulting optimal solution $\xi^{o}$ satisfies

$$
J^{u}\left(\xi^{u}, d^{u}\right) \geq J^{f}\left(\xi^{f}, d^{o}\right) \geq J^{f}\left(\xi^{o}, d^{o}\right) .
$$

Hence, $\xi^{o}$ is also optimal for the UP.

\section{REFERENCES}

[1] Q. Zhou and J. Bialek, "Generation curtailment to manage voltage constraints in distribution networks," IET Generation, Transmission \& Distribution, vol. 1, 2007.

[2] A. Botterud, J. Wang, C. Moteiro, and V. Miranda, "Wind power forecasting and electricity market operations," in Proc. of the IAEE Int. Conf., 2009.

[3] J. Zhou, "20\% wind generation and the energy markets: A model and simulation of the effect of wind on the optimal energy portfolio," Master's thesis, Princeton University, Dep. of Operations Research and Financial Engineering, 2010.

[4] T. Boehme, G. P. Harrison, and A. R. Wallace, "Assessment of distribution network limits for non-firm connection of renewable generation," IET Renewable Power Generation, vol. 4, 2009.

[5] M. H. Albadi and E. F. El-Saadany, "Comparitive study on impacts of wind profiles on thermal units scheduling costs," IET Renewable Power Generation, vol. 5, 2011.

[6] P. P. Varaiya, F. F. Wu, and J. W. Bialek, "Smart operation of smart grid: Risk-limiting dispatch," Proc. the IEEE, vol. 99, 2011.

[7] F. Bouffard and F. D. Galiana, "Stochastic security for operations planning with significant wind power generation," IEEE Trans. on Power Systems, vol. 23, 2008.

[8] J. M. Morales, A. J. Conejo, K. Liu, and J. Zhong, "Real time economic dispatch," IEEE Trans. on Power Systems, 2012.

[9] B. Kirby, "Frequency regulation basics and trends," Oak Ridge National Laboratory, Tech. Rep. ORNL/TM 2004/291, 2004.

[10] J. Sun and L. Tesfatsion, "DC optimal power flow formulation and solution using QuadProgJ,” Iowa State University, Tech. Rep., 2010.

[11] E. Janeček and P. Prautsch, "Closed form estimation of boundary values in electrical distribution networks," in Proceedings of the 16th IFAC World Congress, 2006.

[12] T. Alvey, D. Goodwin, X. Ma, D. Streiffert, and D. Sun, "A securityconstrained bid-clearing system for the New Zealand wholesale electricity market," IEEE Trans. on Power Delivery, vol. 13, 1998.

[13] E. Litvinov, T. Zheng, G. Rosenwald, and P. Shamsollahi, "Marginal loss modeling in LMP calculation," IEEE Trans. on Power Systems, vol. 19, no. 2, 2004.

[14] J. Lavaei and S. H. Low, "Zero duality gap in optimal power flow problem," IEEE Trans. on Power Systems, 2012.

[15] O. Alsac and B. Stott, "Optimal load flow with steady-state security," IEEE Trans. on Power Apparatus and Systems, vol. PAS-93, no. 3, 1974.

[16] R. W. Ferrero, S. M. Shahidehpour, and V. C. Ramesh, "Transaction analysis in deregulated power systems using game theory," IEEE Trans. on Power Systems, vol. 12, no. 3, 1997. 